\title{
Kinks bounded by fermions
}

\author{
Ilya Perapechka \\ Department of Theoretical Physics and Astrophysics, BSU, Minsk 220004, Belarus \\ Yakov Shnir॰ \\ BLTP, JINR, Dubna 141980, Moscow Region, Russia \\ Department of Theoretical Physics, Tomsk State Pedagogical University, Tomsk 634061, Russia
}

(Received 24 October 2019; published 28 January 2020)

\begin{abstract}
We present and study a new mechanism of interaction between solitons based on the exchange interaction mediated by the localized fermion states. As particular examples, we consider solutions of simple $(1+1)$-dimensional scalar field theories with self-interaction potentials, including the sine-Gordon model and the polynomial $\phi^{4}$ and $\phi^{6}$ models, coupled to the Dirac fermions with a backreaction. We discover that there is an additional fermion exchange interaction between the solitons, and it leads to the formation of static multisoliton bound states. Furthermore, we argue that similar mechanisms of formation of stable coupled multisoliton configurations can be observed for a wide class of physical systems.
\end{abstract}

DOI: 10.1103/PhysRevD.101.021701

\section{INTRODUCTION}

One of the most interesting features of the topological solitons, like kinks, vortices, or monopoles (see, e.g., [1,2]), is the remarkable relation between the topological charge of the configuration and the number of fermionic zero modes localized on a soliton. The fundamental Atiyah-PatodiSinger index theorem requires one normalizable fermionic zero mode per unit topological charge [3]. Moreover, apart from zero modes, most configurations support the existence of a tower of localized fermionic modes with nonzero energy.

The fermion zero modes of solitons have been studied for many decades; these states localized on the vortices were discussed first in Ref. [4]. There has been substantial interest in the study of these localized states in various dimensions; examples are fermion modes of the kinks [5-8], monopoles [9,10], sphalerons [11,12], and skyrmions [13-16]. The existence of localized fermions leads to many interesting and unusual phenomena such as fermion number fractionization [6,17], monopole catalysis [9,10], and string superconductivity in cosmology [18] or in chiral superconductors [19].

Fermion zero modes naturally appear in supersymmetric theories; several simple examples are the $N=1$ chiral scalar superfield in $1+1$ dimensions [20] or supersymmetric extensions of the $\mathrm{O}(3)$ nonlinear sigma model [21] and baby-Skyrme model [22,23]. In such a case, the fermion

Published by the American Physical Society under the terms of the Creative Commons Attribution 4.0 International license. Further distribution of this work must maintain attribution to the author(s) and the published article's title, journal citation, and DOI. Funded by SCOAP ${ }^{3}$. zero mode is generated via supersymmetry transformations of the boson field of the static soliton. The breaking of supersymmetry of the configurations, in agreement with the index theorem, shows a spectral flow of the eigenvalues of the Dirac operator with some number of normalizable bounded modes crossing zero.

The typical assumption in most such considerations is that the spinor field does not backreact on the soliton [5-7,24]; moreover, only the fermion zero modes were considered in most cases. A completely different approach to the problem was proposed in our previous works [25-27], where we reconsidered this problem consistently. We found that the backreaction of the localized fermions significantly modifies both the spectral flow and the structure of the coupled configurations. In particular, it results in deformations of the solitons, which to a certain extent resemble the excitations of the internal modes of the scalar configurations [27-29].

In contrast to previous studies, our focus here is not to consider localized fermion states but rather to investigate the pattern of interactions between the solitons in the presence of the additional exchange interaction mediated by localized fermions with finite energy. Our aim is to study the collective fermion modes in the system of kinks in $(1+1)$-dimensional scalar field theories, including the sine-Gordon model and the polynomial $\phi^{4}$ and $\phi^{6}$ models, coupled to the Dirac fermions with a backreaction. Notably, solitons in all these models may support the fermion zero mode, which does not affect the kink for any values of the Yukawa coupling. Furthermore, a single $\phi^{4}$ kink or a kink in the sine-Gordon model [30] may bound other localized fermion modes with finite energy; the number of these modes extracted from the positive and negative continuum increases as the Yukawa coupling becomes stronger. 
The novel aspect is that an exponentially localized fermion bound state may appear in the system of kinks, just in the same way as the collective scalar bound states become trapped by the kink-antikink potential in the $\phi^{6}$ model [31].

In this paper, we investigate multisoliton-fermion systems with a backreaction numerically and elucidate the mechanism for the appearance of the collective fermionic modes. Our computations reveal sequences of new bounded collective fermion states in various systems of solitons. Furthermore, we show that these localized modes give rise to additional interaction between the solitons.

\section{THE MODEL}

The $(1+1)$-dimensional field theory we are interested in is defined by the following Lagrangian:

$$
\mathcal{L}=\frac{1}{2} \partial_{\mu} \phi \partial^{\mu} \phi+\bar{\psi}\left[i \gamma^{\mu} \partial_{\mu}-m-g \phi\right] \psi-U(\phi),
$$

where $U(\phi)$ is a potential of the self-interacting real scalar field $\phi, \psi$ is a two-component Dirac spinor, and $m$ and $g$ are the bare mass of the fermions and the Yukawa coupling constant, respectively. ${ }^{1}$ The matrices $\gamma_{\mu}$ are $\gamma_{0}=\sigma_{1}$ and $\gamma_{1}=i \sigma_{3}$, where $\sigma_{i}$ are the Pauli matrices, and $\bar{\psi}=\psi^{\dagger} \gamma^{0}$. The sine-Gordon (SG) model corresponds to the periodic potential $U(\phi)=1-\cos \phi$ with an infinite number of degenerate vacua $\phi_{0}=2 \pi n, n \in \mathbb{Z}$, the $\phi^{4}$ model corresponds to the quartic potential $U(\phi)=\frac{1}{2}\left(1-\phi^{2}\right)^{2}$ with two vacua $\phi_{0} \in\{-1,1\}$, and the $\phi^{6}$ theory is defined by $U(\phi)=\frac{1}{2} \phi^{2}\left(1-\phi^{2}\right)^{2}$ with triple degenerated vacuum $\phi_{0} \in\{-1,0,1\}$.

Using the standard parametrization for a two-component spinor

$$
\psi=e^{-i \epsilon t}\left(\begin{array}{l}
u(x) \\
v(x)
\end{array}\right)
$$

we obtain the following coupled system of dynamical equations:

$$
\begin{aligned}
\phi_{x x}+2 g u v-U^{\prime} & =0 ; \\
u_{x}+(m+g \phi) u & =\epsilon v ; \\
-v_{x}+(m+g \phi) v & =\epsilon u .
\end{aligned}
$$

This system is supplemented by the normalization condition $\int_{-\infty}^{\infty} d x\left(u^{2}+v^{2}\right)=1$, which we impose as a constraint on the system (2).

For all previously mentioned potentials, in the decoupled limit $g=0$, the model (1) supports spatially localized static topological solitons, the kinks:

\footnotetext{
${ }^{1}$ Here we are using rescaled dimensionless variables.
}

$$
\begin{aligned}
& \Phi_{S G}=4 \arctan e^{x}, \quad \Phi_{\phi^{4}}=\tanh (x), \\
& \Phi_{\phi^{6}}= \pm \sqrt{\frac{1+\tanh x}{2}} .
\end{aligned}
$$

The spectrum of linearized perturbations of the kinks always includes a translational zero mode $\Phi(x) \rightarrow$ $\Phi\left(x-x_{0}\right)$ and the continuum modes (e.g., see $\left.[2,28]\right)$. A peculiarity of the $\phi^{4}$ model is that, apart from these modes, there is also an internal bosonic mode of the kink $\Phi_{\phi^{4}}$.

The coupled pair of the first-order differential equations in (2) over the background provided by the kinks (3) can be transformed into two decoupled Schrödinger-type equations for the spinor components $u$ and $v$ [5]:

$$
\begin{aligned}
& -u_{x x}+\left((m+g \Phi)^{2}-g \Phi_{x}\right) u=\epsilon^{2} u \\
& -v_{x x}+\left((m+g \Phi)^{2}+g \Phi_{x}\right) v=\epsilon^{2} v .
\end{aligned}
$$

We can easily see that the $\phi^{4}$ potential supports exponentially localized fermion zero mode $\epsilon_{0}=0$, up to some modifications of the coupling term [30] or the potential; such a mode exists for all types of the configurations above. The zero eigenvalue does not depend on the Yukawa coupling $g$, and there is no level crossing spectral flow in one spatial dimension. In the special case of the $N=1$ supersymmetric generalization of the model (1) [20], this mode is generated via supersymmetric transformation of the translational mode of the static kink.

One of the essential features of the symmetric $\phi^{4}$ kink is that, for large values of the Yukawa coupling, other localized fermionic states with nonzero energy eigenvalues $|\varepsilon|<|g-m|$ appear in the spectrum [5-8,27,30].

The consideration of the fermion modes bounded to a soliton usually invokes a simplifying assumption that the backreaction of the localized fermions is negligible [5-7,24]. However, coupling to the higher localized modes may significantly distort the $\phi^{4} \mathrm{kink}$; its profile deforms as a fermion occupies an energy level [27]. Furthermore, since such exponentially localized fermion modes may occur in multisoliton systems, localized fermions could mediate the exchange interaction between the solitons. In particular, they may appear as a collective state trapped by the kink-antikink pair $[7,30]$, in a way analogous to the appearance of the collective bosonic modes in the $\phi^{6}$ model [31].

\section{NUMERICAL RESULTS}

To find a numerical solution of the complete system of integral-differential equation (2) with the normalization condition on the spinor field, we used the eighth-order finite-difference method. The system of equations is discretized on a uniform grid with the usual size of 5000 points. For the sake of simplicity, we considered the fermions with zero bare mass $m=0$. The emerging system of nonlinear algebraic equations is solved using a modified 
Newton method. The underlying linear system is solved with the Intel MKL PARDISO sparse direct solver. The errors are on the order of $10^{-9}$.

First, we consider a $K \bar{K}$ pair in the $\phi^{4}$ model with fermions, taking into account the backreaction of the localized modes. Note that in the decoupled limit $g=0$ the $K \bar{K}$ pair is not a solution of the field equations; there is an attractive interaction between the kink and the antikink, and the only solution of the $\phi^{4}$ model in the topologically trivial sector is the vacuum $\phi_{0}=0$. Numerical computations show that, as the Yukawa coupling increases slightly above zero, a nontopological soliton emerges in the scalar sector; this lump is linked to a localized fermionic mode extracted from the positive continuum. As $g$ increases further, the lump becomes larger, and its top corresponds to the maximum of the fermion density distribution. Solutions of that type on the background of a wellseparated approximated kink-antikink pair were discussed in Ref. [7]. We will refer to the modes of that type to as $L_{k}$ modes, where the index $k$ corresponds to the minimal number of nodes of the components.

Taking into account the backreaction of the fermions, we found another, lower-energy branch of solutions. It also emerges from the positive energy continuum, and corresponding solutions represent a well-separated $K \bar{K}$ pair with two fermion modes bounded to each soliton. At finite separation, the spinor field represents a symmetric linear combination of two localized quasizero modes. A further increase of the coupling yields a stronger attraction between the constituents, and the fermion density distribution possess two peaks along this branch.

Furthermore, we observe that in the range of values of the coupling $0<g<1$ there are only quasizero collective modes. As we increased the Yukawa coupling further, we found a sequence of higher collective states bounded by the $K \bar{K}$ pair; see Fig. 1. Similar to the modes localized on the $\phi^{4}$ kink, the first such mode $L_{1}$ appears at $g>1$, the mode $L_{3}$ is extracted from the negative continuum at $g>3$, etc. Evidently, for strong coupling, the backreaction of the fermions cannot be neglected.

Since fermion bound states exist on a single $\phi^{4}$ kink, all collective modes localized on the $K \bar{K}$ pair represent various linear combinations of these states. On the contrary, the model (1), for a given choice of the Yukawa coupling, does not support fermion states bounded either to a single SG kink or to a single $\phi^{6}$ kink. However, the situation can be different in the presence of two or more solitons. Indeed, we found collective fermions localized on various multikink configurations.

The pattern we observed in the SG model is similar yet different from the situation above. Again, a lump coupled to a localized nodeless fermion state $L_{0}$ emerges in the topologically trivial scalar sector, as the Yukawa coupling increases above zero; see Fig. 2. As the coupling gradually increases, the profile of the scalar field becomes
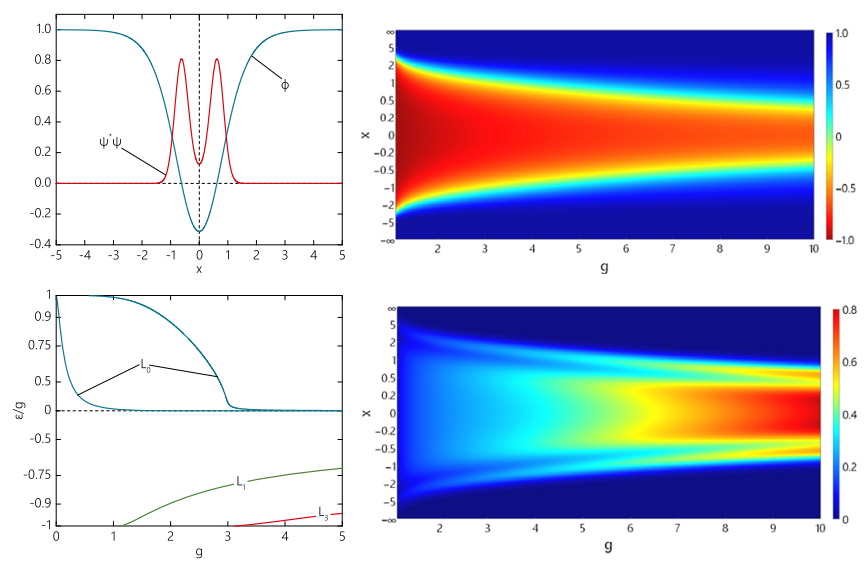

FIG. 1. $\phi^{4}$ kink-antikink pair bounded by fermions. Profiles of the scalar field and fermion density distribution of the collective mode $L_{1}$ at $g=1$ (upper left), normalized energy $\epsilon / g$ of the localized fermionic states as a function of the Yukawa coupling $g$ for several modes (lower left), scalar field of the configuration bounded to the mode $L_{1}$ (upper right), and fermionic density distribution of the mode $L_{1}$ (bottom right) vs Yukawa coupling $g$.

more pronounced, and it approaches the shape of a "frozen" SG breather. At some critical upper value of the Yukawa coupling, this lower in energy branch bifurcates with the second higher in energy branch, which extends all the way backwards to the limit of zero coupling, as seen in Fig. 2. The kinks separate along this branch, while a single fermion mode $L_{0}$ remains at the center of the configuration; it dissolves as the kinks become infinitely separated.

A novel feature of the SG system with backreacting fermions is that a new type of solutions $L_{1}$ emerges at the
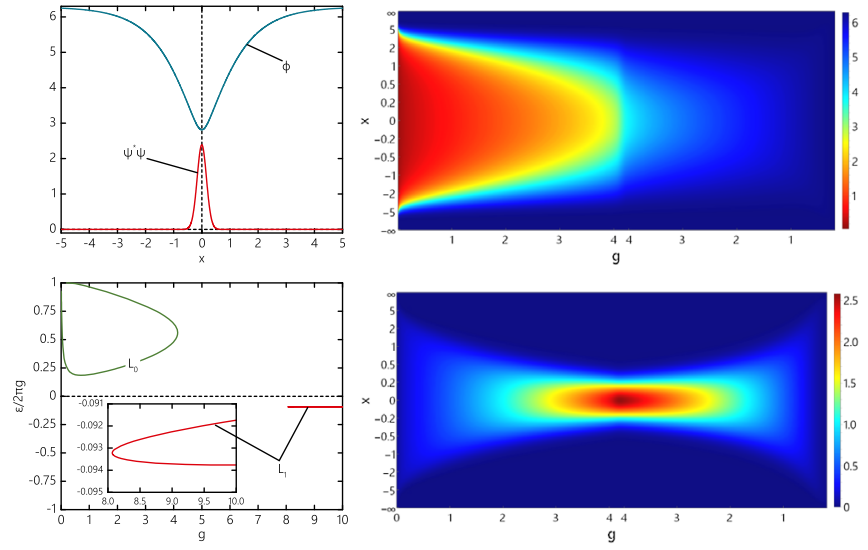

FIG. 2. SG kink-antikink pair bounded by fermions. Profiles of the scalar field and fermion density distribution of the collective mode $L_{0}$ at $g=1$ (upper left), normalized energy $\epsilon / g$ of the localized fermionic states as a function of the Yukawa coupling $g$ for modes $L_{0}$ and $L_{1}$ (bottom left), scalar field of the configuration bounded to the mode $L_{0}$ (upper right), and fermionic density distribution of the mode $L_{0}$ (bottom right) vs Yukawa coupling $g$. 

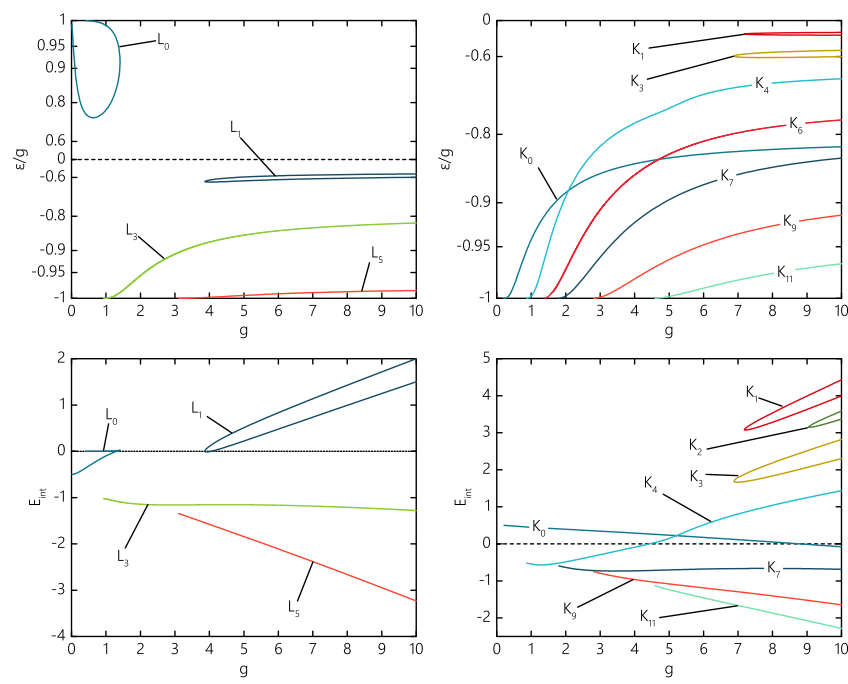

FIG. 3. Fermion states bounded on the $\phi^{6}$ kink-antikink pairs: normalized energy $\epsilon / g$ of the localized modes and the interaction energy $E_{\mathrm{int}}$ as functions of the Yukawa coupling $g$.

bifurcation point $g>8$; see Fig. 2. The corresponding two branches are not linked to the continuum, and a further increase of the Yukawa coupling along both branches yields a strong distortion of the profile of the $K \bar{K}$ configuration which tends toward a deformed $2 K-2 \bar{K}$ coupled system. Physically, the branch structure emerges because of the existence of two linear combinations of the fermion modes, localized on each component.

Indeed, a tower of localized fermion modes also exists on double SG kinks in the sector of topological degree 2. The attractive interaction mediated by the fermions couples the solitons together, and the corresponding spectral flow is not very different from the similar results for a single $S G$ kink in a model with shifted Yukawa interaction [30], although the effects of backreactions become significant for the strong coupling; cf. a similar observation for the $\phi^{4}$ model [27].

Now consider localized fermions in the $\phi^{6}$ model. For different multikink configurations, we found a variety of collective localized modes. First, by analogy with the consideration above, we consider kink-antikink pairs in the topologically trivial sector. Notably, there are two distinct classes of vacua, and they correspond to the $K \bar{K}$ or $(0,1)+(1,0)$ pair and $\bar{K} K$ pair $(1,0)+(0,1)[31]$. Considering the latter configuration, we found two branches of localized fermions linked to the positive continuum; these modes closely resemble similar nodeless collective modes $L_{0}$ on the $K \bar{K}$ pair in the SG model. Higher modes, like $L_{3}$, also appear in the spectrum at large values of the coupling; see Fig. 3. Another analogy with the collective modes $L_{1}$ localized on the $K \bar{K}$ pair in the SG model is that in the $\phi^{6}$ theory this mode is also disconnected from the continuum. On the contrary, a collective potential for the fermions in the presence of the $K \bar{K}$ pair has
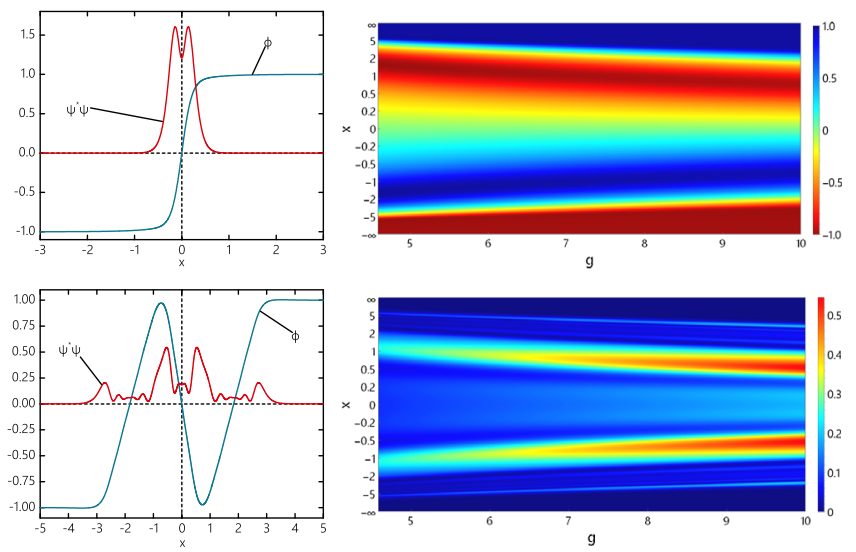

FIG. 4. $\phi^{6}$ multikink configurations bounded by fermions. Profiles of the scalar field and fermion density distribution of the collective mode $K_{0}$ at $g=1$ (upper left) and the chain mode $K_{11}$ at $g=7$ (bottom left), the scalar field of the chain of the kinks $(-1,1)+(1,-1)+(-1,1)$ bounded to the mode $K_{11}$ (upper right), and fermionic density distribution of this configuration (bottom right) as functions of the Yukawa coupling $g$.

a raised central plateau; it does not support fermion bound states. ${ }^{2}$

We found another family of solutions $K_{n}$, and it represents two-kink configurations $(-1,0)+(0,1)$ bounded by collective fermion modes with a minimal node number $n$. Figure 4 shows examples of such states. More fermion bound states do appear for $g>1$; in particular, the modes $K_{1}$ and $K_{3}$ do not emerge from the continuum but appear at some bifurcation point. On the contrary, the modes $K_{2}$ and $K_{4}$ and others are linked to the negative energy continuum; see Fig. 3.

A novel feature of the $\phi^{6}$ system with a backreaction is that it supports an even more complicated bounded multisoliton configuration which represents kink-antikink chains with localized fermion modes. As an example, Fig. 4 exhibits the chain of the kinks $(-1,1)+(1,-1)+(-1,1)$ bounded to the mode $K_{11}$.

The stability of the solutions can be tested by evaluation of the binding energy of the configurations, which we define as the difference between the energy of the scalar components without fermions $E_{0}$ together with the continuum threshold energy $g$ and the total energy of the system: $E_{\text {int }}=E_{0}+g-|\epsilon|-E_{\phi}$. The results of our calculations are displayed in Fig. 3, bottom row. Clearly, some configurations may become unstable with an increase of the coupling $g$.

\section{CONCLUSIONS}

In summary, we have shown that localization of the backreacting fermion modes on multisolitons gives rise

\footnotetext{
${ }^{2}$ Interestingly, there is a certain similarity with the spectrum of linear scalar perturbations in the $\phi^{6}$ model [31].
} 
to a new mechanism of interaction enabling bound multisoliton solutions to occur. More generally, we developed a new method of construction of selfconsistent solutions of the coupled system of integraldifferential equations for a multisoliton configuration coupled to fermions with a backreaction. This method is a powerful tool to study various systems in a wide class of physical systems. There might be a plethora of other applications including fermions on domain walls, cosmic strings, or vortices in the Abelian Higgs model and monopole catalysis. We hope to address these questions in the near future.

\section{ACKNOWLEDGEMENTS}

We are grateful to Vladislav Klimashonok, Steffen Krusch, Tomasz Romańczukiewicz and Andrzej Wereszczyński for enlightening discussions. Ya. S. gratefully acknowledges the support of the Ministry of Science and Higher Education of Russian Federation, Project No. 3.1386.2017.
[1] N. S. Manton and P. Sutcliffe, Topological Solitons (Cambridge University Press, Cambridge, England, 2004).

[2] Y. M. Shnir, Topological and Non-Topological Solitons in Scalar Field Theories (Cambridge University Press, Cambridge, England, 2018).

[3] M. F. Atiyah, V. K. Patodi, and I. M. Singer, Math. Proc. Cambridge Philos. Soc. 77, 43 (1975).

[4] C. Caroli, P. G. De Gennes, and J. Matricon, Phys. Lett. 9, 307 (1964).

[5] R. F. Dashen, B. Hasslacher, and A. Neveu, Phys. Rev. D 10, 4130 (1974).

[6] R. Jackiw and C. Rebbi, Phys. Rev. D 13, 3398 (1976).

[7] Y.Z. Chu and T. Vachaspati, Phys. Rev. D 77, 025006 (2008).

[8] Y. X. Liu, L. D. Zhang, L. J. Zhang, and Y. S. Duan, Phys. Rev. D 78, 065025 (2008).

[9] V. A. Rubakov, Nucl. Phys. B203, 311 (1982).

[10] C. G. Callan, Jr., Phys. Rev. D 26, 2058 (1982).

[11] C. R. Nohl, Phys. Rev. D 12, 1840 (1975).

[12] J. Boguta and J. Kunz, Phys. Lett. 154B, 407 (1985).

[13] J. R. Hiller and T. F. Jordan, Phys. Rev. D 34, 1176 (1986).

[14] S. Kahana, G. Ripka, and V. Soni, Nucl. Phys. A415, 351 (1984).

[15] S. Kahana and G. Ripka, Nucl. Phys. A429, 462 (1984).

[16] G. Ripka and S. Kahana, Phys. Lett. 155B, 327 (1985).
[17] R. Jackiw and P. Rossi, Nucl. Phys. B190, 681 (1981).

[18] E. Witten, Nucl. Phys. B249, 557 (1985).

[19] G. E. Volovik, Pis'ma Zh. Eksp. Teor. Fiz. 70, 601 (1999) [JETP Lett. 70, 609 (1999)].

[20] P. Di Vecchia and S. Ferrara, Nucl. Phys. B130, 93 (1977).

[21] V. A. Novikov, M. A. Shifman, A. I. Vainshtein, and V. I. Zakharov, Fiz. Elem. Chastits At. Yadra 17, 472 (1986) [Phys. Rep. 116, 103 (1984)] [Sov. J. Part. Nucl. 17, 204 (1986)].

[22] C. Adam, J. M. Queiruga, J. Sanchez-Guillen, and A. Wereszczynski, Phys. Rev. D 84, 025008 (2011).

[23] J. M. Queiruga, Phys. Rev. D 94, 065022 (2016).

[24] G. Gibbons, K. I. Maeda, and Y. I. Takamizu, Phys. Lett. B 647, 1 (2007).

[25] I. Perapechka, N. Sawado, and Y. Shnir, J. High Energy Phys. 10 (2018) 081.

[26] I. Perapechka and Y. Shnir, Phys. Rev. D 99, 125001 (2019).

[27] V. Klimashonok, I. Perapechka, and Y. Shnir, Phys. Rev. D 100, 105003 (2019).

[28] N. S. Manton and H. Merabet, Nonlinearity 10, 3 (1997).

[29] N. S. Manton, K. Oles, and A. Wereszczynski, J. High Energy Phys. 10 (2019) 086.

[30] Y. Brihaye and T. Delsate, Phys. Rev. D 78, 025014 (2008).

[31] P. Dorey, K. Mersh, T. Romanczukiewicz, and Y. Shnir, Phys. Rev. Lett. 107, 091602 (2011). 